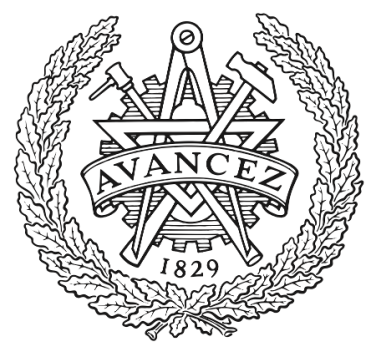

CHALMERS

UNIVERSITY OF TECHNOLOGY

\title{
Can Automotive Radars Form Vehicular Networks?
}

Downloaded from: https://research.chalmers.se, 2023-04-26 11:14 UTC

Citation for the original published paper (version of record):

Aydogdu, C., Wymeersch, H., Rydstrom, M. (2020). Can Automotive Radars Form Vehicular Networks?. IEEE National Radar Conference - Proceedings: 1-6.

http://dx.doi.org/10.1109/RadarConf2043947.2020.9266540

N.B. When citing this work, cite the original published paper. 


\title{
Can Automotive Radars Form Vehicular Networks?
}

\author{
Canan Aydogdu*, Henk Wymeersch*, Mats Rydström ${ }^{\dagger}$ \\ *Department of Electrical Engineering, Chalmers University of Technology, Gothenburg, Sweden \\ ${ }^{\dagger}$ Veoneer Sweden AB, Gothenburg, Sweden \\ Email: \{canan,henkw\}@chalmers.se, mats.rydstrom.external@ veoneer.com
}

\begin{abstract}
Radar communications (RadCom) is a spectrally efficient way for removing automotive radar interference and thereby enhancing reliable radar sensing, via a single hardware for both radar and communications. When interference coordination does not use all the RadCom resources, opportunities for communicating additional data arise. We propose a new communication protocol, termed RadNet (for radar network), which forms a vehicular ad-hoc multi-hop network by automotive radars in a distributed manner. Simulation results obtained for high-way use cases show that RadNet can enable several Mbps data links without degrading the radar performance.
\end{abstract}

\section{INTRODUCTION}

Vehicular networking is a necessity for future intelligent transportation systems (ITS) to enhance safety beyond the range of automotive sensors and beyond the human driver capabilities. Several safety-critical applications such as dissemination of warning messages (future collision warning, blind spot warning, braking ahead warning [1]) and vehicular cooperation for enhancing sensor accuracy or detection of security threats depend on feasibility of a vehicular networking technology.

There are currently two options for connecting vehicles: either via the cellular infrastructure (e.g., 4G Long-Term Evolution for Vehicle (LTE-V) or 5G) or through short-range solutions, such as the WiFi-based IEEE 802.11p used in Dedicated Short-Range Communications (DSRC) in the USA and ITSG5 in Europe [2]. Vehicular networking through the cellular infrastructure is suitable for Internet of Things (IoT) like applications, such as long-term traffic information (e.g., route suggestions), whereas short-range is specifically dedicated to form vehicular ad-hoc networks (VANETs) that provide low-latency transmissions whereas moving the dependency on infrastructure. Recently, a third option for vehicular networking has been proposed, whereby communications occurs via automotive radars that implement radar communications (RadCom) [3]. Vehicular networks can use for example, the unlicensed $60 \mathrm{GHz}$ or so-called mmWave band $(57-66 \mathrm{GHz})$ for dual purpose under restrictions in terms of power emissions [4], [5]; or the 76-81 GHz radar band together with a separate suitable communication channel. When multiple vehicles use such RadCom technology to connect a network of vehicles, we obtain a radar network (RadNet), which is the natural result of convergence of the mm-Wave frequency bands used by wireless communications and automotive radars. RadNets can

978-1-7281-8942-0/20/\$31.00 (C)2020 IEEE

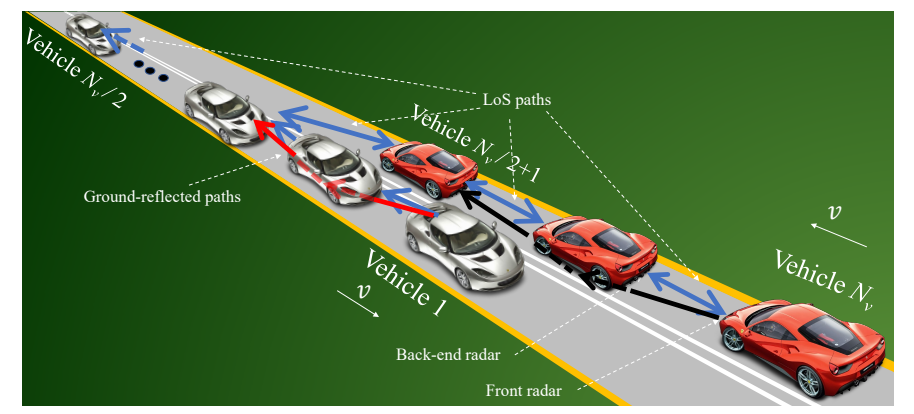

Fig. 1. An illustration of a scenario with example propagation paths of mutual radar interference and communication links, including LOS and ground reflected links.

be used for short-range communications as well as integrated into the mm-Wave cellular communication infrastructure. Although the concept of 'radar networks' was used for integrated radars for environmental mapping or cooperative sensing in military or weather-forecasting [6], it was not considered as an alternative for vehicular communications [2]. Possible forms of co-existence and co-design of automotive radar sensing and cellular communications reviewed in [7] and a radar communications unit employing OFDM for short-range vehicular communications proposed in [8] provide several examples for communication over automotive radars.

There are three distinguished features of a RadNet, which deserves a thorough investigation to find out its suitability for a vehicular networking option. First of all, a highly reliable and low-latency communication system is a missing part of the puzzle of the safe and efficient transportation vision [9]. If a RadNet is formed by replacing radars with radar communication units (RCU) in the next generation of Advanced Driver Assistance (ADAS) and Autonomous Drive (AD) vehicles, V2V communication beams will become directed and more reliable (due to less collisions and increased SINR) and will have lower latency (due to reduced dual function single-onchip processing delays and reduced delays in the wireless channel). Secondly, improved road [10] and parking capacity [11] expected from a future ITS, can only be achieved through high-sensitive localization and the inherent automotive radar sensors offer high localization sensitivity (e.g., up to $3 \mathrm{~cm}$ for $76-81 \mathrm{GHz}$ operating radars), together with robustness against a variety of conditions like snow/fog/rain [12] or optical illusions. Finally, compared to the existing vehicular networking options, a RadNet is more efficient in various aspects: i) 


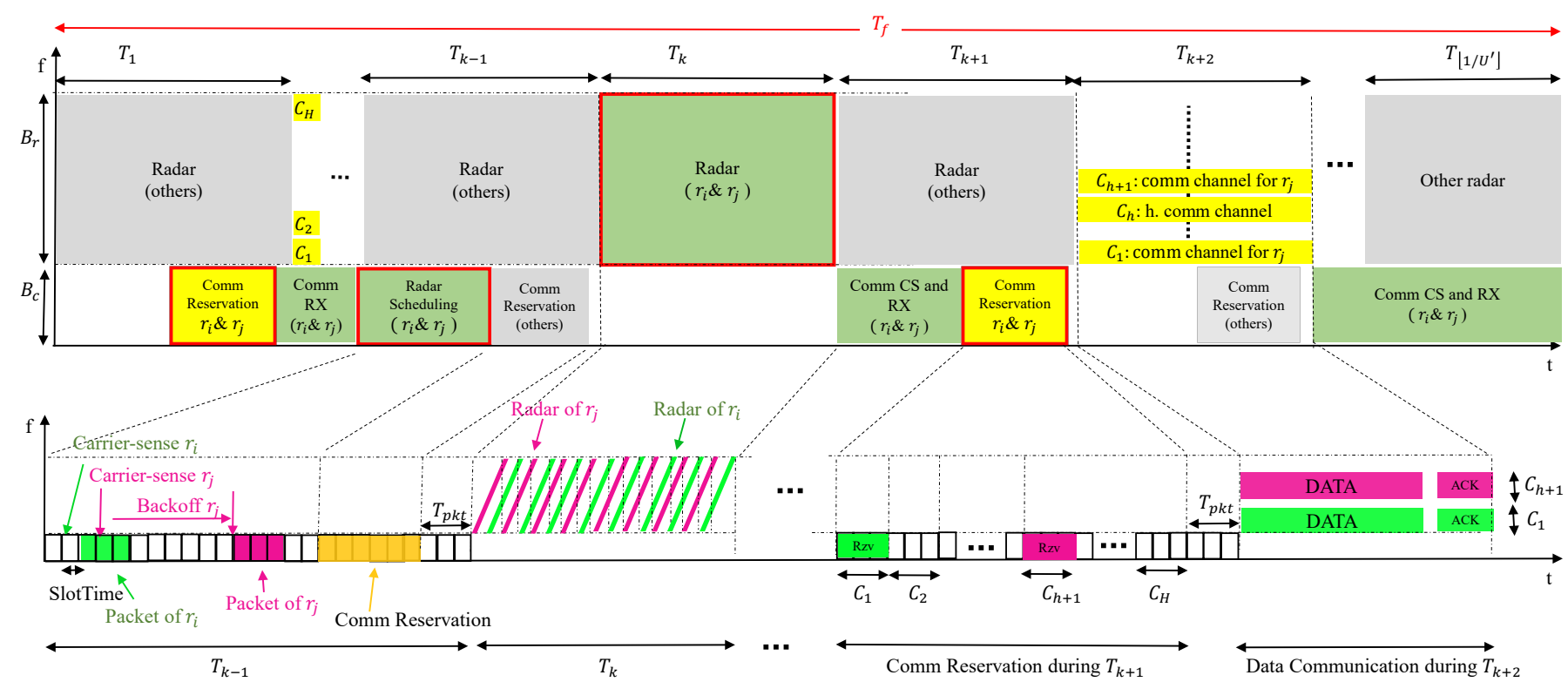

Fig. 2. Illustrates the utilization of frequency-time resources for the RadNet protocol w.r.t. two specific RCUs $r_{i}$ and $r_{j}$. Scheduled radars by RadChat occur over green colored resources, whereas data communications occur over yellow colored resources.

spectrum-efficient, since the underutilized automotive radar spectrum is used by communications; ii) energy-efficient: two functions combined in one SoC; iii) hardware-efficient, since the hardware in a vehicle is reduced from two separate systems to a single dual function hardware.

In this paper, we propose RadNet as a vehicular ad-hoc multi-hop networking technology and investigate its communication capabilities in terms of data rate for high-way traffic. We build on top of our earlier work on the basic communication protocol, RadChat protocol, which coordinates radar transmissions in order to address the mutual interference problem [13], [14], leading to reduced ghost targets [15] and improved pedestrian detections [16]. We show that, cooperative radar communications can also be used to achieve data communication among vehicles on the order of tens of Mbps with a reasonable latency besides eliminating mutual radar interference.

\section{SySTEM MODEL}

All automotive radars are assumed to be replaced with RCUs, which have an additional communication plus interference mitigation capability, such as the RadChat RCUs [14]. RCUs thus have both the automotive radar and communication capability within a single hardware and are controlled via a central unit in each vehicle. And radar transmission is considered successful if it is received without any in-band interference.

All vehicles use FMCW-type radars with the same bandwidth $B_{\mathrm{r}}$, same chirp duration $T$, and same frame time $T_{f}$, comprising $N$ chirps per frame.

RCUs switch between radar and communication functionality. However, interference-free radar sensing is always prioritized over communication. In order to achieve this, the total system bandwidth is divided in band $B_{\mathrm{c}}$ used for control packets (communication reservation and radar scheduling packets), and in band $B_{\mathrm{r}}$ radar transmissions occur, as well as data communication for external applications (such as warning messages, see-through driving data, sensor fusion data).

Two types of communication packets are used: control and data packets. Control packets are unacknowledged broadcast messages by RadChat for interference mitigation and are sent over a separate frequency band $B_{\mathrm{c}}$, which lies out of the radar sensing frequency band $B_{\mathrm{r}}$. Data packets are used for vehicular communication, such as warning messages, seethrough driving data, sensor fusion data, and are sent over frequency bands within $B_{\mathrm{r}}$ if this band is free from radar sensing.

\section{RAdNet: Protocol Description}

RadNet is a distributed vehicular networking protocol based on cooperative FMCW-based radar communications. The goal of RadNet is to provide data communication, such as distributing warning messages, radar map data, see-through driving data, etc., in a mutual radar interference-free channel. Interference-free radar sensing is always prioritized over communication in a RadNet; and communication takes place only when the radar sensor becomes idle during a radar frame. In this section, we describe the proposed RadNet protocol.

\section{A. Division in Time Slots}

Time division is a necessary feature for the operation of RadNet for enabling cooperative radar communications since the same hardware is used for both functionalities. Fig. 2 illustrates the utilization of frequency-time resources for the RadNet protocol w.r.t. two specific RCUs $r_{i}$ and $r_{j}$. The RCUs $r_{i}$ and $r_{j}$ transmit radar signals during time slot $T_{k}$, where a 


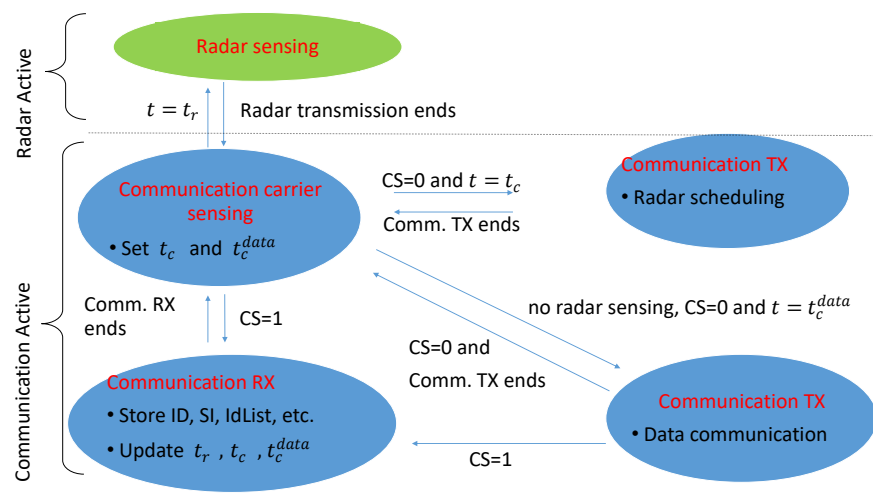

Fig. 3. Flowchart for RadNet operation.

time slot is a portion of a periodically repeated radar frame. We divide a radar frame $T_{f}$ into $\left\lfloor 1 / U^{\prime}\right\rfloor$ time slots, where $U^{\prime}=(N+1) T / T_{f}$, corresponding to a modified duty cycle with one extra chirp added to a chirp sequence in order to allocate more than one radar sequence to the same time slot. This slotted time within a radar frame is set to maximize the non-overlapping chirp sequences within a radar frame. Other RCUs in the VANET are allowed to transmit their FMCW chirp sequences during other time slots, except $T_{k}$, over the band $B_{r}$ in Fig. 2 (colored in grey). Communication control packet transmission over the band $B_{c}$ is also adjusted according to these time slots. For example, the RCUs $r_{i}$ and $r_{j}$ do send broadcast control packets for mutual radar interference-free operation, i.e., RadChat, during $T_{k-1}$.

This division in time results in a certain maximum number of RCUs with no mutual interference in the vehicular network, which is denoted by $M_{\max } . M_{\max }$ is the maximum size of the disjoint time-frequency resources that a radar can be scheduled at for interference-free vehicular communication. Note that a maximum of $\left\lfloor B_{\mathrm{r}} /\left(\left(1+\alpha_{d}\right) B_{\mathrm{c}}\right)\right\rfloor$ radars can be sent during one time slot without interfering each other, where $\alpha_{d}$ is a constant for determining the maximum range of a direct interferer taken into account [14]. Hence, $M_{\max } \leq\left\lfloor 1 / U^{\prime}\right\rfloor\left\lfloor B_{\mathrm{r}} /\left(\left(1+\alpha_{d}\right) B_{\mathrm{c}}\right)\right\rfloor$.

\section{B. Services Provided}

The RadNet protocol provides two services:

- Radar scheduling service: mutual radar interference is mitigated by the RadChat protocol [14], by scheduling radars to non-interfering time-frequency resources.

- Data communication service: vehicular data communication takes place during unused time-frequency resources in the band $B_{\mathrm{r}}$, without compromising radar performance.

These services are implemented on top of a single RadCom hardware using different medium access control (MAC) techniques described next. A flowchart for the operation of RadNet is given in Fig. 3, where $t_{r}$ is the starting time of radar transmission, $t_{c}$ is starting time of communication control packet for radar scheduling and $t_{c}^{\text {data }}$ is the starting time of data packet.
1) Radar Scheduling Service: In order to provide interference-free automotive radar sensing, RCUs should transmit their chirp sequences in non-overlapping time slots over the radar band $B_{\mathrm{r}}$ and non-conflicting vulnerable periods within a time slot [15]. The value of such resources is called as SlotIndex (SI), where SIs range from 1 to $M_{\max }$. Hence, each RCU needs to schedule its radar transmission in a specific SI, which should be different than the SIs employed by other vehicles. The RadChat protocol [14] explained next, provides this in a distributed manner over both single-hop and multihop VANETs.

Prior to radar chirp sequence transmission, each RCU broadcasts a packet over a separate communication channel with carrier sense multiple access (CSMA) with random binary exponential backoff (BEB). For example, let the RCU $r_{i}$ have a radar chirp sequence transmission during $T_{k}$ in Fig. 2 within the radar frame $T_{f}$. This RCU broadcasts a control packet during $T_{k-1}$ (green-coloured rectangle on the bottom left corner). Let us assume that another RCU $r_{j}$ receives this control packet. The control packet includes the identity (ID: the reference time employed), the SlotIndex (SI: randomly selected index for one of the disjoint time-frequency resources) and the idList (the list of vehicles using the same ID). RCU $r_{j}$, which plans to radar sense during $T_{k}$, does not send an acknowledgement, but updates its own ID, SI and idList according to this received control packet, so that its radar transmission is scheduled to a different and possibly nonconflicting SI. Any conflicts or collisions of control packets are resolved in other radar frames since BEB is employed. The radar chirp sequences of RCU $r_{j}$ lie in $T_{k}$ but use a nonconflicting vulnerable period after these adjustments. RCU $r_{j}$ broadcasts its radar information by a control packet during $T_{k-1}$ (pink-coloured rectangle on the bottom left corner).

As a result, RadChat avoids mutual radar interference by scheduling radar sensing to non-overlapping vulnerable periods, avoids communication packet collisions by CSMA with BEB for communications, while radar and communication cross-interferences are avoided by using a different communication band than radar (refer to [14] and [17] for a detailed explanation of single-hop and multi-hop operation of RadChat).

2) Data communication service: This service is an extension to the radar scheduling service and constitutes the primary contribution of this article. The goal of data communication service of RadNet is to coordinate data communication, which is achieved by a reservation-based random MAC technique. The RCUs, which radar sense during $T_{k}$, do carrier-sense the control channel starting $T_{k+1}$. If no activity is sensed during $T_{k+1}$ in the control channel, these RCUs are allowed to utilize a portion of the radar band $B_{r}$ during the time slot $T_{k+2}$ (and also other time slots except $T_{k-1}$ and $T_{k}$ ) with data communication. The radar band is divided into data communication channels $C_{h}$, for $1 \leq h \leq H_{\max }$, where $H_{\max }=\left\lfloor B_{\mathrm{r}} / B_{\mathrm{c}}\right\rfloor$ is the maximum number of available data communication channels that don't mutually overlap. For example, the RCUs $r_{i}$ and $r_{j}$ carrier-sense and receive packets 
over the control channel as long as radar is inactive in order to cope with dynamic vehicular topologies during the green coloured durations over the control band in Fig. 2. If no radar scheduling takes place during $T_{k+1}$ in the control channel, a communication reservation interval in the control band is used for allocation of the data communication channels (indicated by yellow in the right bottom of Fig. 2). An RCU willing to transmit data to one or more destinations sends a ready-tosend (RTS) packet during a randomly selected reservation slot, say $C_{h} \in T_{k+1}$. The recipient RCUs tune their RF circuitry to the corresponding channel $C_{h}$ in the next time slot $T_{k+2}$. Data is transmitted during $T_{k+2}$ over channel $C_{h}$, which is acknowledged. This way, RadNet provides an acknowledged connectionless service, with either unicast or multicast transmissions depending on the intended destinations.

\section{Random versus Packed Assignment}

Since we prioritize radar, data communication can never take place if radar sensing occurs at every time slot in a radar frame. In our previous studies [14], [15], [17], radars are scheduled randomly to the available slot indices (SIs). We call this Random Assignment (RA). However, it is possible to assign radar sensing to one or few time slots, i.e., packing radar sensing in time, which we call as Packed Assignment $(P A)$. After convergence of RadChat, a packed assignment of SIs can be distributed over the control packets to vehicles, either controlled by a clusterhead RCU or in a distributed manner. The details of the protocol for PA are omitted for space reasons.

\section{Performance Evaluation And Results}

\section{A. Evaluation Scenario}

Two high-way traffic scenarios are investigated, where one fleet of vehicles moves in the same direction in ScenarioI and two fleets of vehicles move in opposite directions in Scenario-II (see Fig. 4). A geometry-based deterministic vehicular channel model is employed owing to high-frequency

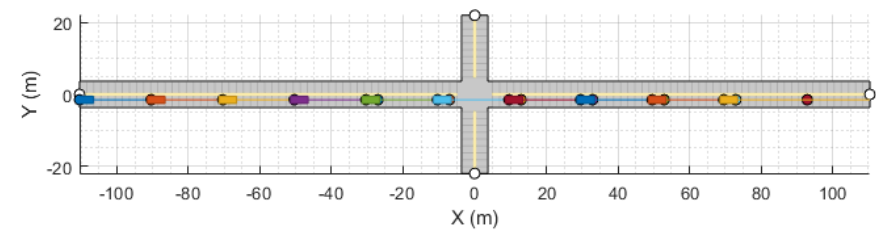

(a)

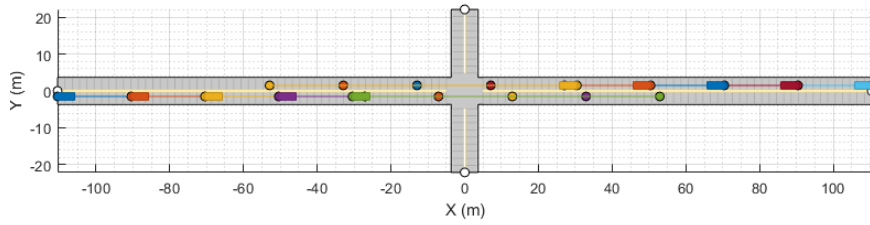

(b)

Fig. 4. Traffic scenarios: a) Scenario-I, one fleet moving in one direction and b) Scenario-II, two fleets approaching each other. Vehicles are represented by rectangles, whereas waypoints, i.e., starting and ending paths, of each vehicle is indicated by dots with same color.
TABLE I

SIMULATION PARAMETERS [14]

\begin{tabular}{|c|c|c|}
\hline & Parameter & Value \\
\hline \multirow{11}{*}{$\underset{\tilde{z}}{\tilde{z}}$} & Radar bandwidth $\left(B_{\mathrm{r}}\right)$ & $800 \mathrm{MHz}$ \\
\hline & ADC bandwidth $\left(B_{\mathrm{ADC}}\right)$ & $15 \mathrm{MHz}$ \\
\hline & Carrier frequency $\left(f_{\mathrm{r}}\right)$ & $79.15 \mathrm{GHz}$ \\
\hline & Modified duty cycle $\left(U^{\prime}\right)$ & $1 / 5$ \\
\hline & Vehicle radar cross sections $\sigma$ & $10 \mathrm{dBsm}$ \\
\hline & Radar transmit power $P_{\mathrm{r}}$ & $12 \mathrm{dBm}$ \\
\hline & Radar processing gain $G_{p}$ & $53.76 \mathrm{~dB}$ \\
\hline & Radar signal to noise ratio threshold $\gamma_{\mathrm{r}}$ & $7 \mathrm{~dB}$ \\
\hline & Chirp duration $(T)$ & $77.51 \mu \mathrm{s}$ \\
\hline & Frame duration $\left(T_{f}\right)$ & $50 \mathrm{~ms}$ \\
\hline & Number of chirps per frame $(N)$ & 129 \\
\hline \multirow{8}{*}{ 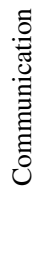 } & Communication bandwidth $B_{\mathrm{C}}$ & $15 \mathrm{MHz}$ \\
\hline & Communication carrier frequency $\left(f_{\mathrm{C}}\right)$ & $79.02 \mathrm{GHz}$ \\
\hline & Packet size $\left(N_{\text {pkt }}\right)$ & 100 bytes \\
\hline & Modulation & 16-QAM \\
\hline & Communication transmit power $P_{\mathrm{C}}$ & $23 \mathrm{dBm}$ \\
\hline & SlotTime $\delta$ & $10 \mu \mathrm{s}$ \\
\hline & Maximum contention window size $\left(W_{0}\right)$ & 48 \\
\hline & Maximum backoff stage $(B)$ & 3 \\
\hline \multirow{6}{*}{$\stackrel{\mathscr{\Xi}}{\stackrel{\Xi}{\circ}}$} & Maximum range of LoS interference & $1 \mathrm{~km}\left(\alpha_{d}=6.56\right)$ \\
\hline & Thermal noise temperature $T_{0}$ & $290 \mathrm{~K}$ \\
\hline & Receiver's noise figure & 2 \\
\hline & Antenna gain $G_{\operatorname{trx}}$ & $30 \mathrm{dBi}$ \\
\hline & Reflection Coefficient for asphalt $(R)$ & 0.2814 \\
\hline & Antenna FoV & $\begin{array}{l} \pm 10^{\circ} \text { (azimuth), } \\
\pm 5^{\circ} \text { (elevation) }\end{array}$ \\
\hline
\end{tabular}

mmWave bands [18], [19] and we assume that signals propagate either through a LoS path if not blocked, or through ground-reflections. The signal to noise ratios (SNR) perceived at RCUs are computed using Friis free space propagation and radar equations [20]. The values of $\alpha_{d}$ is assigned so that the maximum range of a direct interferer taken into account is set as $1 \mathrm{~km}$. Communication links with a data rate equal to $2 B_{c}$ are assumed to be available if a bit error rate of at most $10^{-5}$ is achieved for the 16-QAM signals over the AWGN channel.

We ignore interference among radars within a single vehicle since each vehicle is assumed to be equipped with one frontend and one back-end RCU with long-range radar sensing functionality. All vehicles are assumed to be equally spaced and have the same speed. The achievable data rate performance of the distributed cooperation-based radar communications protocol RadNet is evaluated in two dynamic VANET fleets scenarios for $20 \mathrm{~m}$ vehicle spacing and speeds of $v=$ $150 \mathrm{~km} / \mathrm{h}$, which provides us a communication performance at a regime where we push the limits of automotive radar performance at reasonable maximum relative velocities. A total of 10 Monte Carlo simulations of $2 \mathrm{~s}$ duration were performed, with the parameters summarized in Table I.

\section{B. Discussion}

Scenario-I (one fleet): We first evaluate the impact of the number of vehicles in the fleet on the convergence time of RadChat (denoted by $t_{\text {final }}$ ) and the achieved rate upon convergence of RadChat. The data rate per vehicle versus the convergence time of interference mitigation for Scenario-I is 


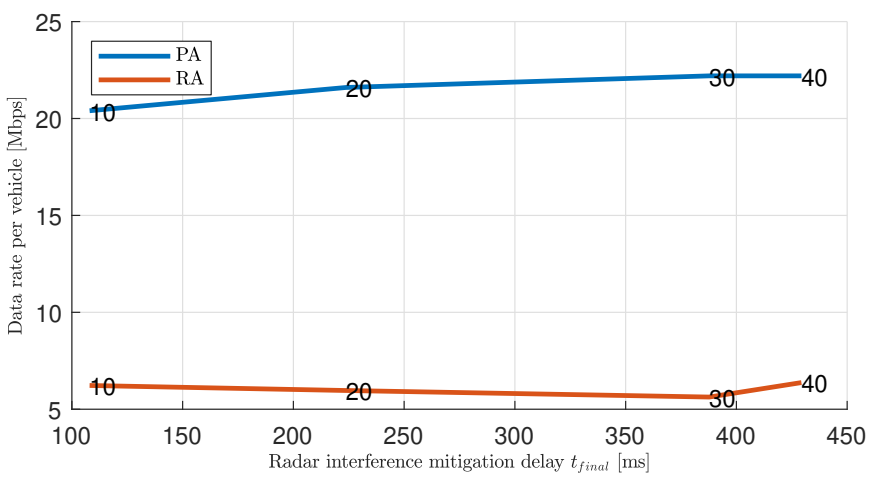

Fig. 5. Scenario-I performance for the radar interference mitigation convergence time $t_{\text {final }}$ versus communication data rate per vehicle for random assignment (RA) and packed assignment (PA).

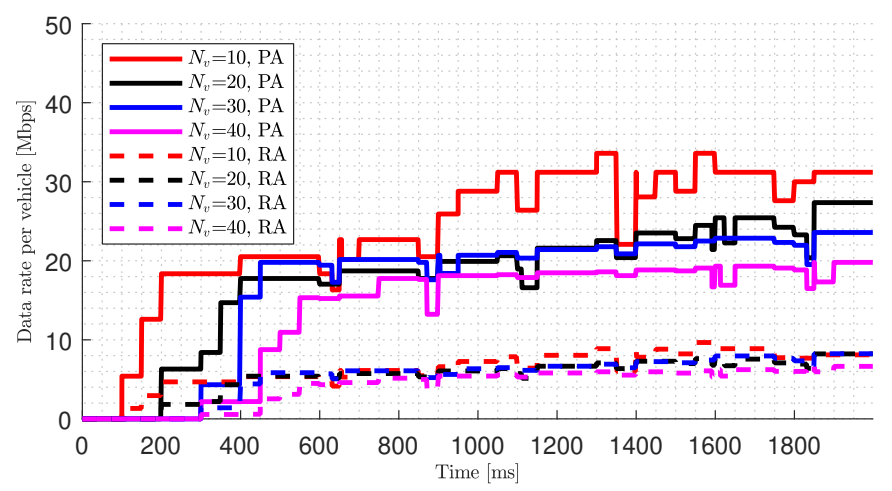

(a)

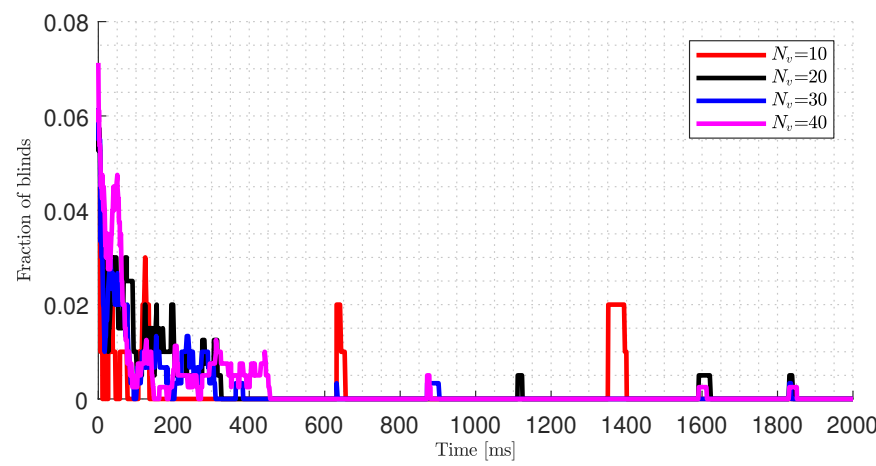

(b)

Fig. 6. Scenario-II performance for $N_{v}=\{10,20,30,40\}$ : a) communication data rate with random assignment (RA) and packed assignment (PA) with RadChat and b) fraction of radar blinds.

given in Fig. 5 for various number of vehicles $N_{v}$ denoted on the curves.

Note that each vehicle is connected to up to 4 other vehicles, 2 through LOS links and 2 through ground-reflected links. Hence, the number of communication links is linear with the number of vehicles, which makes data rate per vehicle almost constant. It can be observed that RA provides about 6.05 Mbps, whereas PA provides 21.6 Mbps data rate per vehicle on the average. This suggests that a RadNet provides data

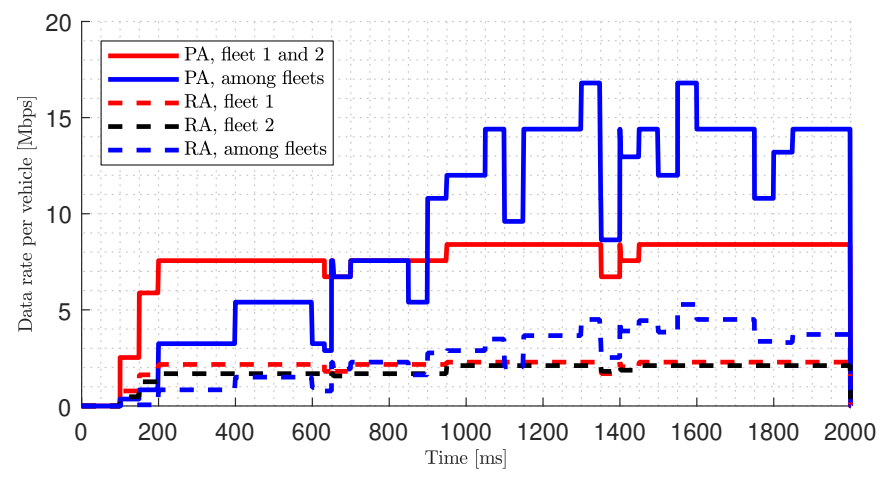

(a)

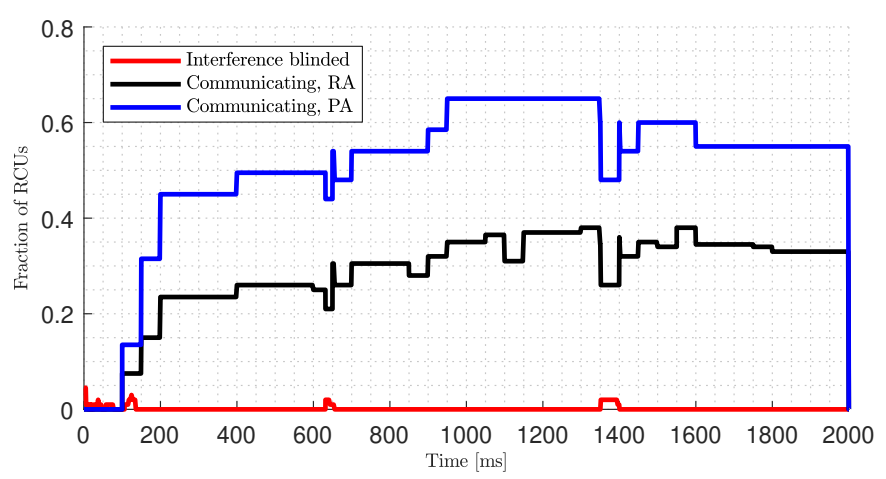

(b)

Fig. 7. Scenario-II performance for $N_{v}=10$ vehicles: a) communication data rate, b) fraction of blind and communicating RCUs with random assignment (RA) and packed assignment (PA) with RadChat.

rates comparable to DSRC by reusing radar spectrum during radar idle times. These rates are enough for dissemination of warning messages, while see-through driving data might be relayed by doubling the radar ADC bandwidth for the considered fleet of vehicles on a high-way scenario.

Scenario-II (two approaching fleets): For two approaching fleets, the network topology will change continuously, so the achieved rate varies over time. For that reason we evaluate communication and radar performance as a function of time, for a varying number of vehicles. Fig.6-a) shows the data rate per vehicle. The data rate starts off low, as the RadChat protocol takes most resources to coordinate radar interference (this takes about 100-400 ms), after which the rate increases. The rates are higher than in Scenario-I, since a vehicle may connect to more surrounding vehicles via $\operatorname{LoS}$ or ground reflection. As in Scenario-I, the packed assignment leads to rates about 3 to 4 times higher than under random assignment. The radar performance in shown in Fig.6-b), in terms of the fraction of radar blinds (i.e., non-functioning due to interference) as a function of time. Observe the correlation between increased blinds and reductions in rate. Again, the convergence of RadChat is visible within $400 \mathrm{~ms}$, with small adjustments around 600 and $1400 \mathrm{~ms}$, due to the changing topology. Note that RadChat operates in a distributed manner and each individual vehicle experiences a different convergence latency 
which is lower than the maximum value reported here. Hence, RadChat convergence latency is determined by the number of neighboring RCUs rather than the number of RCUs in the whole VANET. as shown in Fig. 6(b).

When two fleets approach, there is both intra-fleet and interfleet communication. In order to understand the rates for each type of communication, Fig. 7-a) depicts the data rate per vehicle within each fleet and between fleets, for $N_{v}=10$, while Fig. 7-b) shows the fraction of communicating vehicles, as well as the fraction of radar blinds. We see that up to $60 \%$ of vehicles are able to send data with almost $15 \mathrm{Mbps}$ across a fleet moving with a $300 \mathrm{~km} / \mathrm{h}$ relative speed with RadNet, whereas the data rate per vehicle within the fleet is almost constant around $8 \mathrm{Mbps}$. About half of the RCUs do communicate while radar sensing is not affected at all.

\section{Conclusion}

When radar hardware is reused for communication purposes, it enables not only interference coordination, but also additional data services. We have proposed a distributed radar networking solution, which we evaluated in a case study to assess the achievable data rate. This solution, named as RadNet, builds on the existing RadChat protocol and provides low radar interference and high-rate communications. Based on realistic radar parameters and a geometric channel model, we achieve data rates comparable to the widespread shortrange vehicular solution DSRC.

\section{ACKNOWLEDGMENT}

This research was supported by Vinnova grant 2018-01929 and the Chalmers Transport Area of Advance project IRIS.

\section{REFERENCES}

[1] K. Sjöberg, P. Andres, T. Buburuzan, and A. Brakemeier, "Cooperative intelligent transport systems in europe: Current deployment status and outlook," IEEE Vehicular Technology Magazine, vol. 12, no. 2, pp. 8997, 2017.

[2] J. Wang, J. Liu, and N. Kato, "Networking and communications in autonomous driving: A survey," IEEE Communications Surveys Tutorials, vol. 21, no. 2, pp. 1243-1274, 2019.

[3] A. R. Chiriyath, B. Paul, and D. W. Bliss, "Radar-communications convergence: Coexistence, cooperation, and co-design," IEEE Transactions on Cognitive Communications and Networking, vol. 3, no. 1, pp. 1-12, March 2017.

[4] T. Yamawaki and S. Yamano, "60-GHz millimeter-wave automotive radar," Fujitsu technical review, vol. 15, no. 2, pp. 9-18, 1998.

[5] P. Kumari, N. Gonzalez-Prelcic, and R. W. Heath, "Investigating the IEEE 802.11ad standard for millimeter wave automotive radar," in IEEE Vehicular Technology Conference, Sept 2015, pp. 1-5.
[6] A. Frischen, J. Hasch, and C. Waldschmidt, "A cooperative mimo radar network using highly integrated fmcw radar sensors," IEEE Transactions on Microwave Theory and Techniques, vol. 65, no. 4, pp. 1355-1366, 2017.

[7] V. Petrov, G. Fodor, J. Kokkoniemi, D. Moltchanov, J. Lehtomäki, S. Andreev, Y. Koucheryavy, M. J. Juntti, and M. Valkama, "On unified vehicular communications and radar sensing in millimeter-wave and low terahertz bands," CoRR, vol. abs/1901.06980, 2019. [Online]. Available: http://arxiv.org/abs/1901.06980

[8] Y. Han, E. Ekici, H. Kremo, and O. Altintas, "Automotive radar and communications sharing of the 79-GHz band," in Proceedings of the First ACM International Workshop on Smart, Autonomous, and Connected Vehicular Systems and Services, ser. CarSys '16. New York, NY, USA: ACM, 2016, pp. 6-13.

[9] M. Boban, A. Kousaridas, K. Manolakis, J. Eichinger, and W. Xu, "Connected roads of the future: Use cases, requirements, and design considerations for vehicle-to-everything communications," IEEE Vehicular Technology Magazine, vol. 13, no. 3, pp. 110-123, Sep. 2018.

[10] "Study on enhancement of 3GPP support for 5G V2X services (release 15)," Tech. Rep., Nov. 2016.

[11] M. Ferreira, L. Damas, H. Conceicão, P. M. d'Orey, R. Fernandes, P. Steenkiste, and P. Gomes, "Self-automated parking lots for autonomous vehicles based on vehicular ad hoc networking," in IEEE Intelligent Vehicles Symposium Proceedings, June 2014, pp. 472-479.

[12] J. Ryde and N. Hillier, "Performance of laser and radar ranging devices in adverse environmental conditions," Journal of Field Robotics, vol. 26 no. 9, pp. 712-727, 2009

[13] C. Aydogdu, M. F. Keskin, G. K. Carvajal, O. Eriksson, H. Hellsten, H. Herbertsson, E. Nilsson, M. Rydstrom, K. Vanas, and H. Wymeersch, "Radar interference mitigation for automated driving: Exploring proactive strategies," IEEE Signal Processing Magazine, vol. 37, no. 4, pp. 72-84, 2020.

[14] C. Aydogdu, M. F. Keskin, N. Garcia, H. Wymeersch, and D. W. Bliss, "RadChat: Spectrum sharing for automotive radar interference mitigation," IEEE Transactions on Intelligent Transportation Systems, pp. 1-14, 2019.

[15] C. Aydogdu, N. Garcia, L. Hammarstrand, and H. Wymeersch, "Radar communication for combating mutual interference of FMCW radars," in IEEE Revolutions in Radar, Boston, USA, 22-26 Apr. 2019.

[16] C. Aydogdu, N. Garcia, and H. Wymeersch, "Improved pedestrian detection under mutual interference by FMCW radar communications," in IEEE International Symposium on Personal, Indoor and Mobile Radio Communications (PIMRC), Workshop on 5G V2X Communications for Connected Autonomous Driving, Sept. 2018.

[17] C. Aydogdu, M. F. Keskin, and H. Wymeersch, "Automotive radar interference mitigation via multi-hop cooperative radar communications," in EuMA European Microwave Association, $17^{\text {th }}$ European Radar Conference EuRad 2020, Utrecht, Netherlands, submitted 2020.

[18] L. Liang, H. Peng, G. Y. Li, and X. Shen, "Vehicular communications: A physical layer perspective," IEEE Transactions on Vehicular Technology, vol. 66, no. 12, pp. 10647-10659, Dec 2017.

[19] W. Viriyasitavat, M. Boban, H. Tsai, and A. Vasilakos, "Vehicular communications: Survey and challenges of channel and propagation models," IEEE Vehicular Technology Magazine, vol. 10, no. 2, pp. 5566, June 2015.

[20] A. Al-Hourani, R. J. Evans, S. Kandeepan, B. Moran, and H. Eltom, "Stochastic geometry methods for modeling automotive radar interference," IEEE Transactions on Intelligent Transportation Systems, vol. 19, no. 2, pp. 333-344, 2017. 